\title{
Análise Comparativa Epidemiológica da Cirurgia de Esofagectomia Realizada por Via Transtorácica e por Via Minimamente Invasiva
}

\section{Comparative and Epidemiological Analysis of Esophagectomy Through Transtoracic and Minimally Invasive Surgery}

Giovanna Vidal Belo ${ }^{\dagger *}$, Júlia Vidal Spinelli, Lívia Soares Viana ${ }^{\dagger}$, Michelle Garcia Ferreira de Oliveira de Souza ${ }^{\dagger}$, Adriana Rodrigues Ferraz

Como citar esse artigo. Belo, G.V;

Spinelli, J.V.; Viana, L.S.; de Oliveira, M.G.F.; de Souza, S.A.; Ferraz, A.R Análise Comparativa Epidemiológica da Cirurgia de Esofagectomia Realizada por Via Transtorácica e por Via Minimamente Invasiva. Revista de Saúde. 2020 Jan./Jun.; 11 (1): 21-24.

\section{Resumo}

Diversas patologias que acometem o esôfago - como tumores, acalasia e megaesôfago - são corrigidas cirurgicamente através da esofagectomia, um procedimento baseado na remoção parcial ou total do esôfago. A esofagectomia distal retira apenas parte do esôfago e existem duas técnicas para sua execução: a esofagectomia com toracotomia (ECT), com abertura da caixa torácica, ou a esofagectomia sem toracotomia (EST), feita através do endoscópio, apresentando vantagens com relação à ECT. O objetivo do estudo foi analisar comparativamente os diversos aspectos epidemiológicos da ECT e EST. Trata-se de um estudo observacional e transversal, a partir de dados coletadas no SIH/SUS entre Janeiro de 2014 a Abril de 2019, analisando número de óbitos e de internações, taxa de mortalidade e valor de cada procedimento em cada região do Brasil, comparando os dados obtidos aos achados bibliográficos. Os dados foram analisados por meio dos testes de T-Student e Correlação de Pearson. De acordo com as informações encontradas, foram realizadas 544 internações, sendo a maioria delas decorrentes de EST, realizadas no regime público. A despeito da menor amostra na ECT, as maiores taxas de mortalidade ocorreram na EST, apesar dessa diferença não ter sido estatisticamente significativa. A média da permanência hospitalar foi menor na ECT, mas a diferença não foi estatisticamente significativa, o que não é compatível com a literatura, que indica recuperação mais rápida na EST. Assim, não houve diferença significante entre os dados estatísticos e epidemiológicos na morbi-mortalidade dos procedimentos, corroborando para escolha do tratamento individualizado de cada paciente.

Palavras-chave: Esofagectomia; Epidemiologia dos Serviços de Saúde; Epidemiologia; Toracotomia; Cirurgia Torácica.

\begin{abstract}
Several pathologies affecting the esophagus, such as tumors, achalasia and megaesophagus, are surgically corrected through esophagectomy, a procedure based on the partial or total removal of the esophagus. Distal esophagectomy removes only part of the esophagus and there are two techniques for its execution: esophagectomy with thoracotomy (ECT), with thoracic cavity opening, or esophagectomy without thoracotomy (EST) performed through the endoscope, presenting advantages over ECT. The objective of the study was to comparatively analyze the different epidemiological aspects of ECT and EST. It is an observational and cross-sectional study, based on data collected in the SIH / SUS between January 2014 and April 2019, analyzing number of deaths and hospitalizations, mortality rate, complexity and value of each procedure in each region of Brazil, comparing the data obtained to the bibliographic findings. Data were analyzed using T-Student test and Pearson's correlation. According to the information found, 544 hospitalizations were performed, most of them due to EST, performed in the public regime. Despite the smallest sample in the ECT, the highest mortality rates occurred in EST, although this difference was not statistically significant. The mean length of hospital stay was lower in ECT, but the difference was not statistically significant, which is not compatible with the literature, that indicates a faster recovery in EST. Thus, there was no significant difference between the statistical and epidemiological data on the morbimortality of the procedures, corroborating the choice of the individualized treatment of each patient.
\end{abstract}

Keywords: Esophagectomy; Health Services Research; Epidemiology; Thoracotomy; Thoracic Surgery.

\section{Introdução}

O câncer de esôfago é, no Brasil, o sexto tipo mais frequente entre homens e o $16^{\circ}$ entre mulheres. Quando se analisam os dados da epidemiologia mundial, trata-se do oitavo mais frequente ${ }^{1}$. Histologicamente há dois tipos de câncer de esôfago: o carcinoma epidermoide, que é o mais frequente e está relacionado a diversos fatores de risco comprovados, e o adenocarcinoma, originado a partir do epitélio metaplásico do esôfago de Barret, considerado oprincipal fator de risco do desenvolvimento cancerígeno ${ }^{2}$. Já o acometimento do esôfago pela doença de Chagas é denominado megaesôfago chagásico, e ocorre devido à lesão dos plexos musculares pelo Trypanosoma cruzi, um protozoário que causa dilatação progressiva deste órgão. Clinicamente, o aspecto mais

\footnotetext{
Afiliação dos autores:

${ }^{\dagger}$ Discentes do Curso de Medicina da Universidade de Vassouras, Vassouras - RJ, Brasil

${ }^{\ddagger}$ Docente do Curso de Medicina da Universidade de Vassouras, Vassouras - RJ, Brasil
} 
marcante é a disfagia progressiva. Acomete, na América Latina, cerca de 8 milhões de pessoas, apresentando uma mortalidade de até 670.000 indivíduos por ano ${ }^{3}$. Diferente do megaesôfago chagásico, que tem um agente causal bem definido, a acalasia do esôfago é um quadro idiopático caracterizado pela redução ou pela ausência completa da peristalse do corpo do esôfago. Além disso, há falha do mecanismo fisiológico de relaxamento do esfíncter esofágico inferior durante a deglutição, o que desencadeia a principal manifestação clínica: a disfagia ${ }^{4}$.É o distúrbio de motilidade esofágica mais comum, apresentando uma incidência de 1 a cada 100.000 pessoas por ano ${ }^{5}$.

Todos os quadros descritos podem ser corrigidos cirurgicamente através da esofagectomia, cirurgia de remoção parcial ou total do esôfago. A esofagectomia distal remove apenas o terço distal do esôfago, e existem duas técnicas distintas utilizadas para essa remoção. A primeira é a esofagectomia com toracotomia (ECT), também denominada transtorácica. Nela, são feitas incisões no abdome e na caixa torácica, e a escolha do tipo de incisão é feita com base em critérios utilizados pelo cirurgião. Já a esofagectomia sem toracotomia (EST), ou minimamente invasiva, é feita com o auxílio de um endoscópio, que monitora o procedimento enquanto este é realizado através de pequenas incisões. Apresenta vantagens como menor perda sanguínea, menor dor pós-operatória, menor tempo de internação hospitalar e recuperação pós-operatória mais rápida ${ }^{6}$. Em ambas as técnicas, é importante estar atento à cadeia linfática comprometida, que deve ser removida e enviada para análise histopatológica, levando em consideração a superioridade já comprovadas do método minimamente invasivo sobre o método com toracotomia em relação à abordagem para a retirada dos linfonodos? ${ }^{7}$.

O objetivo do presente estudo foi analisar, de forma comparativa, diferentes aspectos epidemiológicos das informações existentes sobre as modalidades de esofagectomia supracitadas (aberta com toracotomia e minimamente invasiva sem toracotomia) e avaliar crítica e estatisticamente os dados encontrados.

\section{Materiais e Métodos}

Este estudo teve parecer aprovado de número 3.328. Realizou-se um estudo observacional e transversal, com base em dados obtidos no SIH/DATASUS de procedimentos hospitalares, sobre os procedimentos esofagectomia distal com toracotomia e esofagectomia distal sem toracotomia, abrangendo os meses de Janeiro de 2014 a Abril de 2019. Os dados utilizados incluem número de internações, número de óbitos, taxa de mortalidade, tempo médio de internação, regime dos procedimentos e valor dos serviços hospitalares, correlacionando os achados aos dados disponíveis na literatura.

Após a obtenção dos dados, foi realizada a análise estatística por meio do programa Microsoft Excel, utilizando os seguintes testes estatísticos: Teste de T-Student e Correlação de Pearson.

\section{Resultados}

No período analisado, houve um total de 544 internações para a realização de esofagectomia distal no Brasil, divididos em 264 (48,5\%) ECT e 280 (51,5\%) EST. A região com maior número de procedimentos realizados foi a Região Sudeste, com 109 ECT e 147 EST, seguida pelas regiões Nordeste (respectivamente 47 e 68 procedimentos), Sul (50 e 25), Centro-Oeste (33 e 25$)$ e Norte (25 e 15). Com relação ao regime utilizado, a informação não estava presente em $62,5 \%$ das internações, ainda que, dentre os procedimentos que continham essa informação, o regime mais comum foi o público (164 contra 40 procedimentos em regime privado).

Dentre a amostra utilizada, houve um total de 80 óbitos. Deles, 43 ocorreram nos procedimentos de ECT, sendo 19 óbitos na região Sudeste (representando 17,4\% dos procedimentos realizados na região), 8 na região Centro-Oeste $(24,2 \%), 6$ na região Sul $(12 \%)$ e 5 nas regiões Nordeste $(10,6 \%)$ e Norte $(20 \%)$. Esses dados resultaram em taxas de mortalidade média no país de 16,29 óbitos a cada 1.000 procedimentos, dividida da seguinte forma: taxa de mortalidade de 17,43 na região Sudeste, 24,24 na região Centro-Oeste, 12 na região Sul, 20 na região Norte e 10,64 na região Nordeste. Já na análise dos óbitos ocorridos pós EST, a distribuição geográfica ocorreu da seguinte forma: 21 dos 37 óbitos ocorreram na região Sudeste (representando 14,2\% dos procedimentos realizados na região), seguida de 6 óbitos na região Nordeste $(8,8 \%), 4$ na região Sul $(16 \%)$ e 3 nas regiões Norte $(20 \%)$ e Centro-Oeste $(12 \%)$. Esses números geraram uma taxa de mortalidade no país de 13,21 óbitos a cada 1.000 procedimentos, divididas nas regiões da seguinte forma: 14,29 no Sudeste, 8,82 no Nordeste, 16 na região Sul, 20 na região Norte e 12 na região Centro-Oeste. Vale notar que a comparação entre os procedimentos demonstrou que a diferença entre taxa de mortalidade e número de óbitos entre os dois procedimentos não foi estatisticamente relevante (respectivamente, $\mathrm{p}=0,31$ e $\mathrm{p}=0,81$ ).

Foi analisado, ainda, o tempo médio de permanência hospitalar dos pacientes. A maior média de permanência foi da EST na região Sul, enquanto a menor média de permanência foi a ECT na região Norte. A média de permanência entre as regiões foi de 20,3 dias na ECT e 20,8 dias na EST, o que também não foi estatisticamente significativo $(p=0,29)$. Houve uma fraca correlação positiva entre os valores de tempo 
médio de permanência hospitalar e taxa de mortalidade $(0,40$ na ECT e 0,28 na EST).

Com relação a gastos públicos, foram utilizados um total de R \$ 3.106.076,14 para esses procedimentos, dividido em R \$ 1.751.131,93 na ECT e R \$ 1.354.944,21 na EST. A região com maiores gastos foi o Sudeste, que despendeu quase metade dos gastos do país, com um total de R $\$ 1.539 .475,76$. Ao calcular-se o valor gasto por procedimento, obteve-se uma média de R\$ $5.709,70$ por esofagectomia, sendo que a região na qual o procedimento foi mais caro foi a região Sul (R\$ 6.742,90), e mais barato na região Norte (R\$ $4.359,43)$. Não houve correlação entre o valor gasto por procedimento e a taxa de mortalidade da região $(0,12)$.

\section{Discussão}

A predominância da realização cirúrgica, no Brasil, deu-se no Sudeste, cujos custos também foram os mais dispendiosos dentre as regiões. É provável que haja uma relação do maior acesso aos serviços de mais altas complexidades de qualidade de regiões mais urbanas e industrializadas, como Sudeste, com a maior taxa de cirurgias realizadas e uma menor mortalidade proporcional. No Centro-Oeste, por exemplo, foram realizadas 33 ECT com 8 óbitos decorrentes, representando a maior taxa de mortalidade para a quantidade de procedimentos concluídos $(22,2 \%)$, enquanto no Sudeste, das 109 ECT, 18 vieram a óbito, representando $17,4 \%$.

Apesar do menor número de cirurgias realizadas com toracotomia $(48,5 \%)$, a taxa de mortalidade se mostra discretamente maior $(53,75 \%)$ do que em pacientes operados sem a toracotomia, apesar de esse dado não ter se mostrado estatisticamente relevante. A média de permanência hospitalar, também não foi estatisticamente relevante quando comparados os dois procedimentos, apesar da média ter sido ligeiramente menor na ECT. Esse dado não está de acordo com a literatura disponível, que indica uma recuperação mais rápida em pacientes após EST. Por isso, indica-se estudos mais aprofundados no tema, para evidenciar as causas do achado estatístico em questão.

Em linhas gerais, os diversos dados apresentados não caracterizam significantes diferenças estatísticas de morbi-mortalidade e tempo de internação quando comparados os dados epidemiológicos de ECT e EST.

Deve-se destacar, porém, duas limitações importantes do estudo: a primeira é referente aos métodos utilizados no procedimento. Pelos dados disponíveis na plataforma SIH/DATASUS, não é possível determinar o tipo de técnica cirúrgica utilizado em cada procedimento. Sabe-se, porém, que dentre as duas categorias mais utilizadas para a realização da esofagectomia (anastomose torácica e anastomose cervical), a anastomose cervical possui maior morbimortalidade, devido a maior incidência de fístulas ${ }^{8}$, com elevada letalidade hospitalar relacionada a esta complicação. Além disso, as limitações das pesquisas baseadas em dados obtidos pela plataforma do DATASUS são conhecidas. Apesar de possibilitar informação ampla e acesso livre, estudos têm demonstrado que os dados sofrem grande variabilidade, o que está relacionado, principalmente, a falta de preenchimento padronizado para codificação dos diagnósticos ${ }^{9}$. A despeito disso, a base de dados do DATASUS segue sendo um importante órgão de produção, organização e disseminação de informação que pode ser utilizado desde que conhecidas e reconhecidas as suas deficiências.

\section{Considerações Finais}

A esofagectomia é uma cirurgia que cursa com sensível mudança de hábitos por parte do paciente, trazendo diversas limitações. Os valores apresentados e discutidos corroboram que o tratamento de patologias esofagianas deve sempre ser individualizado, de acordo com as condições médicas locais e as condições individuais de apoio ao paciente. A escolha dentre os procedimentos possíveis deve possibilitar integridade e qualidade de vida, gerando a menor morbimortalidade possível. Cabe ao médico, dessa forma, estabelecer um curso de ação que leve em consideração os benefícios individuais, mais do que as poucas diferenças, que ECT e EST podem oferecer.

\section{Referências}

1. Câncer de esôfago [Internet]. INCA - Instituto Nacional de Câncer. 2018 [cited 8 June 2019]. Available from: https://www.inca.gov.br/tipos-decancer/cancer-de-esofago.

2. Kasper, DL. et al. Medicina interna de Harrison. 19. ed. Porto Alegre: AMGH, 2017.

3. Pochini CD, Gagliardi D, Saad Júnior R, Almeida RF, Corsi PR Esofagectomia com gastroplastia no megaesôfago avançado: análise tardia da importância do uso do omeprazol. Revista do Colégio Brasileiro de Cirurgiões. 2015 Oct;42(5):299-304

4. Aquino JL, Said MM, Pereira DR, AMARAL PC, Lima JC, Leandro-Merhi VA. Surgical treatment analysis of idiopathic esophagealachalasia. ABCD. Arquivos Brasileiros de Cirurgia Digestiva (São Paulo). 2015 Jun;28(2):98101.

5. Júnior JE, Naves BL, Ramos RF, Leite MD. Tratamento cirúrgico de acalasia pela realização de esofagocardiomiotomia com fundo plicaturavideolaparoscópica à Heller-Pinotti em paciente com megaesôfago grau IV: Relato de caso. Rev Med Minas Gerais. 1884; 2017(27).

6. Cola CB, Sabino FD, Pinto CE, Morard MR, Portari FP, Guedes T. Esofagectomia vídeo-tóraco-laparoscópica com tempo torácico em posição pronada. Rev. Col. Bras. Cir. [Internet]. 2017 Oct [cited 2019 June 08]; 44(5): 428-434.

7. Smithers BM, Gotley DC, Martin I, Thomas JM. Comparison of the outcomes between open and minimally invasive esophagectomy. Annals of surgery. 2007 Feb;245(2):232.

8. Tomasich FD, Valladares GC, Demarchi VC, Gagliardi D. Influência 
do local da anastomose (cervical ou torácica) na morbi-mortalidade das esofagectomias. Revista Brasileira de Cancerologia. 2003 Jan; 49(1):47-54.

9. Mathias TA, Soboll ML. Confiabilidade de diagnósticos nos formulários de autorização de internação hospitalar. Revista de Saúde Pública. 1998; 32:526-32. 